\title{
Functional Requirements of Cellular Differentiation: Lessons from Bacillus subtilis
}

Jatin Narula ${ }^{1}$, Masaya Fujita ${ }^{2}$ and Oleg A. Igoshin ${ }^{1 *}$

${ }^{1}$ Department of Bioengineering, Rice University

${ }^{2}$ Department of Biology and Biochemistry, University of Houston

*author to whom correspondence should be addressed: igoshin@rice.edu

\section{Abstract}

Successful execution of differentiation programs requires cells to assess multitudes of internal and external cues and respond with appropriate gene expression programs. Here, we review how Bacillus subtilis sporulation network deals with these tasks focusing on the lessons generalizable to other systems. With feedforward loops controlling both production and activation of downstream transcriptional regulators, cells achieve ultrasensitive threshold-like responses. The arrangement of sporulation network genes on the chromosome and transcriptional feedback loops allow coordination of sporulation decision with DNA-replication. Furthermore, to assess the starvation conditions without sensing specific metabolites, cells respond to changes in their growth rates with increased activity of sporulation master regulator. These design features of the network enable cells to robustly decide between vegetative growth and sporulation.

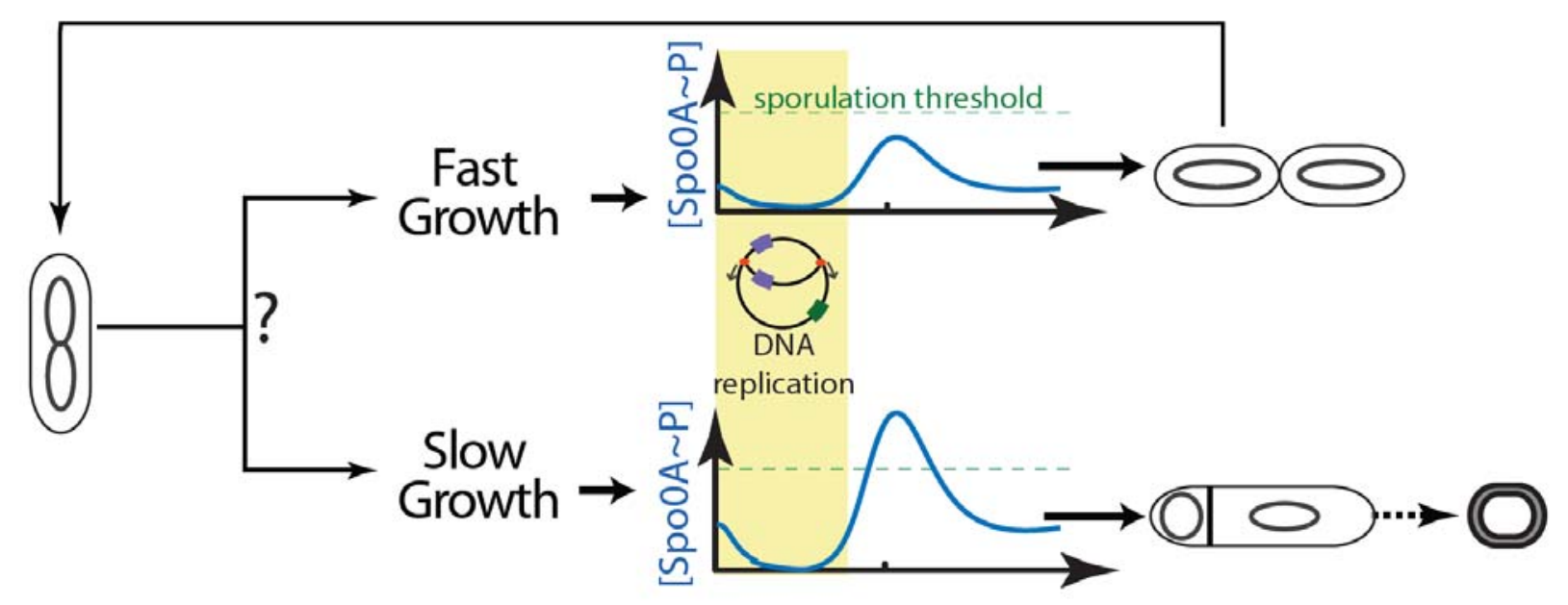




\section{Introduction}

Cellular differentiation is a generic term for the processes by which cells specialize phenotypically and morphologically to adapt to their environmental conditions or to perform specific functions [1]. This ability to generate specialized cell types from multipotent precursor cells is certainly central to the development of multi-cellular eukaryotes [1-3] but it is also ubiquitous in single cell organisms [4-7]. Over decades of prior research, it has become evident that cell differentiation primarily results from specialization of the gene expression repertoire [8-11]. This specialization in turn is controlled by Gene Regulatory Networks (GRNs) that sense changes in the cellular environment, detect and process differentiation and stress related signals and execute the appropriate gene expression response with a combination of transcriptional and post-translational controls [3,12]. Therefore to mechanistically understand cell differentiation programs we have to study the structure and function of the underlying GRNs.

Sporulation in Bacillus subtilis has long been used as a model for prokaryotic differentiation. This differentiation program enables $B$. subtilis cells to produce metabolically inert and stress-resistant spores capable of surviving long periods of starvation (Fig. 1A). From decades of molecular genetic studies, a large and complex network controlling sporulation has been uncovered [5,13,14](Fig. 1B). However, as with most GRNs controlling cellular differentiation (hereafter, differentiation GRNs), from a cursory glance the sporulation network appears an inscrutable mess of interconnected components impeding mechanistic analysis. However, more recent and careful examinations of the network structure show that it is in fact hierarchically organized into functionally distinct modular sub-networks $[13,15]$. At the top of the hierarchy is the phosphorelay - a four component version of the typical bacterial two component system [16]. The phosphorelay comprises histidine kinases (KinA-E), which can auto-phosphorylate in response to environmental signals. These kinases then transfer their phosphoryl groups to the sporulation master regulator Spo0A via phosphotransferases SpoOF and SpoOB. Increase in phosphorylated Spo0A (an active form, hereafter Spo0A P) activates transcription of a multitude of genes, including those encoding two alternative sigma factors $\sigma^{F}$ and $\sigma^{\mathrm{E}}$, and promotes asymmetric division [17]. Subsequently, $\sigma^{F}$ and $\sigma^{E}$ are activated post-translationally in a compartment-specific manner in the forespore and the mother cell respectively (Fig. 1A). Following this break of symmetry, the two compartments initiate their separate gene expression programs eventually resulting in spore formation $[14,17,18]$.

This type of modular organization is not unique to sporulation and is, in fact, a shared structural pattern across a wide range of biological networks. Many differentiation GRNs similarly contain at least three modules (Fig. 1B): (i) A signal-sensing/encoding module (such as the phosphorelay) that senses all the relevant intracellular and extracellular cues for the specific differentiation program and integrates all the information into a level and activity of the master regulator; (ii) One or a few differentiation master regulators (such as SpoOA in B. subtilis) that orchestrate the network response, i.e. encode all the relevant signals and dictate the cell-fate decision; and (iii) signal decoding/execution modules (such as $\sigma^{\mathrm{F}}$ and $\sigma^{\mathrm{E}}$ activation networks) that operate downstream of master regulators and convert the activity level of the master regulator into a go-no-go binary decision whether to activate differentiation-specific genes. 

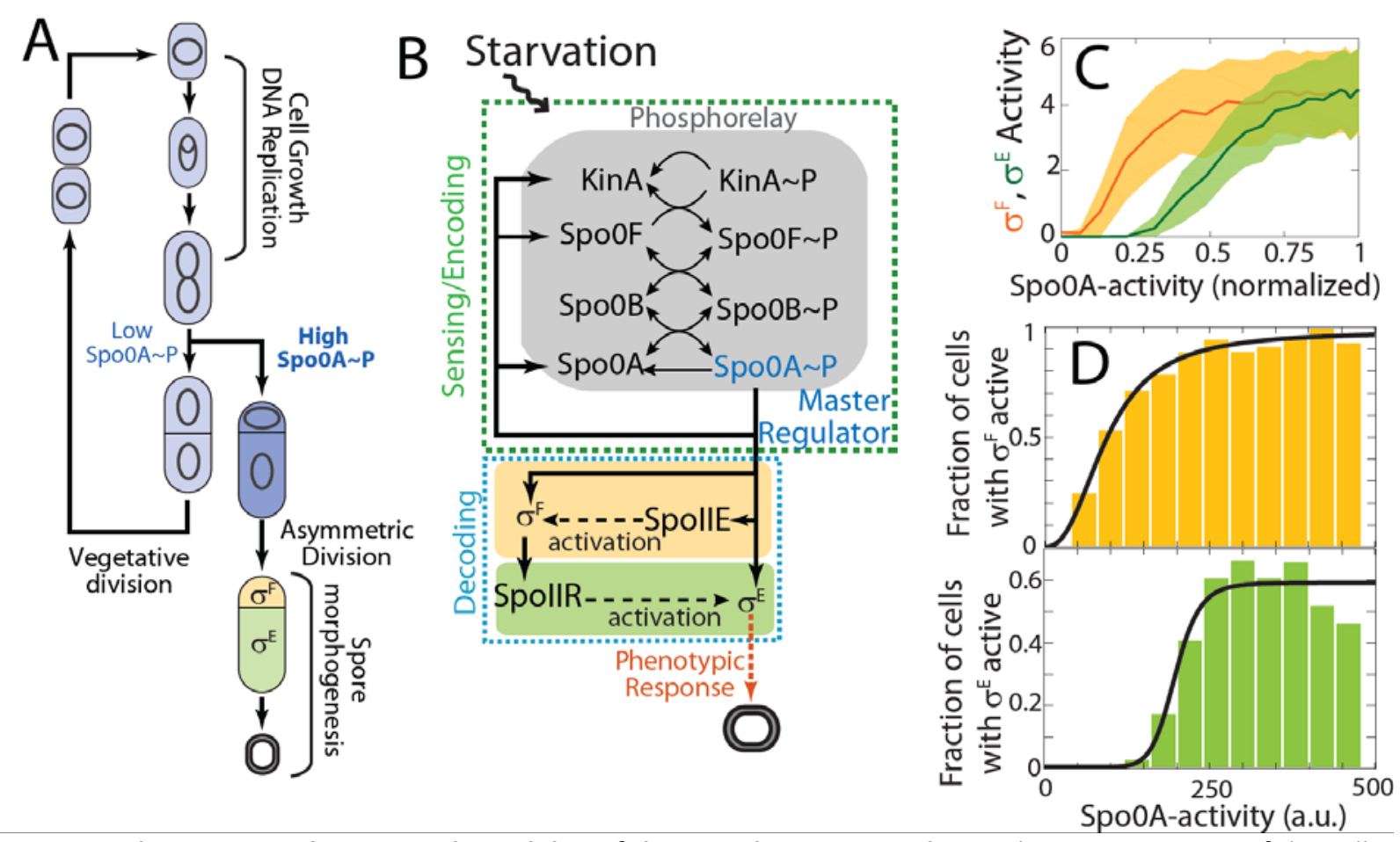

Figure 1. Ultrasensitive decision making ability of the sporulation network. A. Schematic overview of the cellfate decision between vegetative division and sporulation made by $B$. subtilis cells during starvation. B. Diagram of the sporulation network that senses starvation and controls the cell-fate decision. C. Modeling predictions for the responses of $\sigma^{\mathrm{F}}$ and $\sigma^{\mathrm{E}}$ activation. Solid lines and shaded areas represent the means and standard deviations of the response calculated from stochastic simulations. D. Single cell fluorescence measurements of the responses of the $\sigma^{\mathrm{F}}$ and $\sigma^{\mathrm{E}}$ activation modules as a function of SpoOA activity in wildtype cells. A triple reporter strain was used to measure SpoOA and both sigma factors activities simultaneously in wildtype cells grown in starvation media. Cells were binned based on CFP fluorescence for Spo0A activity (PspollA-cfp), and the fractions of cells in each bin that are $\sigma^{\mathrm{F}}$-active and $\sigma^{\mathrm{E}}$-active (based on appropriate PspollQ-mCherry and PspolID-yfp fluorescence thresholds) are shown in the upper and lower panels respectively. The solid lines represent Hill equation fits. These fits indicate that, as predicted by modeling results, fractions of cells displaying both $\sigma^{\mathrm{F}}$ and $\sigma^{\mathrm{E}}$ activities increase ultrasensitively at different SpoOA activity thresholds.

The similarity in structure of different GRNs is unsurprising, since these regulatory networks are the result of evolutionary selective pressures to successfully execute their respective differentiation programs, which often share functional requirements $[2,19,20]$. This structure-function-relationship view suggests that if we parse all the regulatory tasks of a particular differentiation GRN, we should be able to understand the structural design of that enables these tasks. Moreover the similarity of network structures implies that uncovering structure-function relationships for one particular differentiation GRN can yield general design principles - rules relating functional requirements of network with its designs. Such principles can enable our understanding of a GRN structures in the wider context $[19,21]$. In this review, using the $B$. subtilis sporulation network, we demonstrate how a combination of mathematical modeling and quantitative experiments enables understanding of design principles for differentiation GRNs. 


\section{Design principles of Bacillus subtilis sporulation network}

\section{Switch-like gene expression control}

Cell differentiation normally requires the activation of a one set of genes and the down-regulation of another $[8,11]$. Thus the key requirement for a cellular differentiation GRN is the ability to dramatically shift gene expression profiles within a cell. Many studies of GRNs focus specifically on elucidating the mechanisms of this switch-like behavior [22]. Two types of GRN responses are known to achieve this switch-like behavior: ultrasensitive (i.e. highly nonlinear) and bistable (i.e. toggle-switch-like) responses
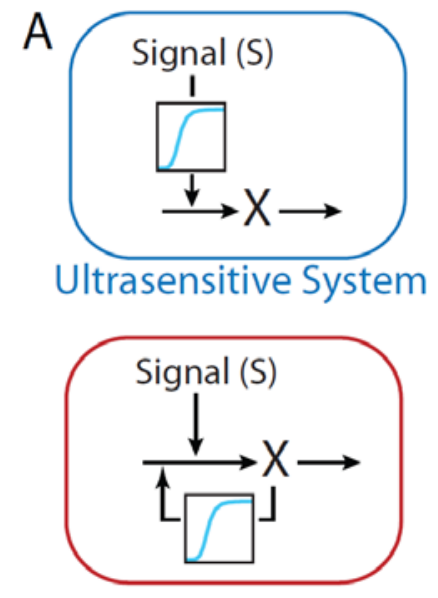

Bistable System

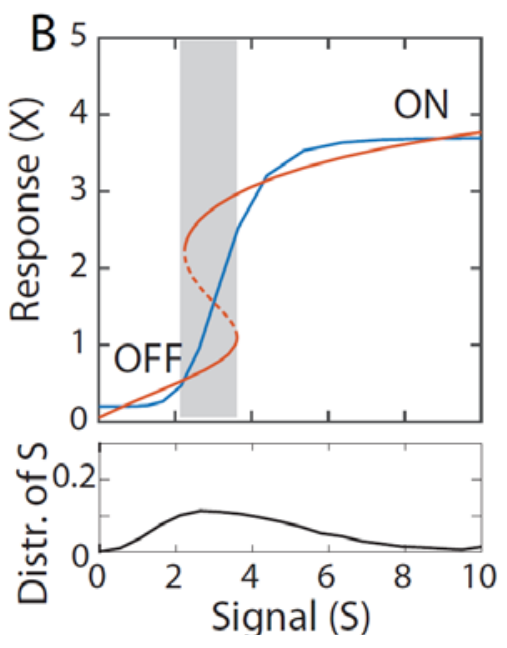

$\mathrm{C}$

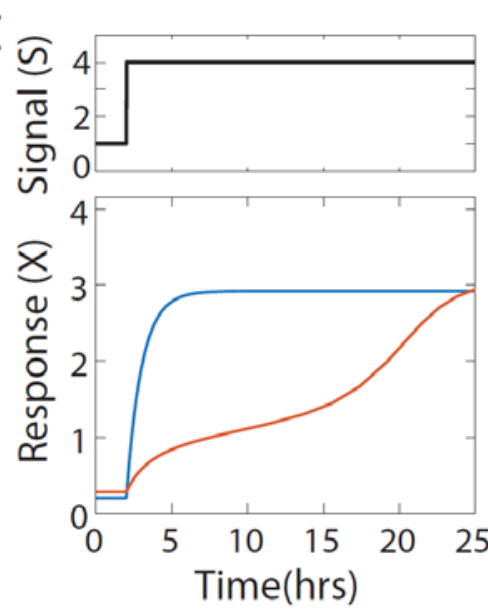

Box 1: Switch-like responses convert small changes in signal (input) to large changes of response (output) and thereby allow cells to achieve distinct gene-expression states. Mechanistically these responses can be divided into one of two types (Panel A): (i) ultrasensitive systems that display a strongly nonlinear(sigmoidal) but continuous response to signal and (ii) bistable systems that have multiple steady states and discontinuously jump between these at a threshold signal level.

Ultrasensitivity can be generated by variety of mechanisms. Among the best characterized mechanisms are allosteric or cooperative enzymes, competing molecular processes operating near saturation (zero-order), multi-site phosphorylation and molecular titration $[24,25,27]$. On the other hand, bistable responses result from nonlinear positive feedback, either explicit (common for gene autoregulation regulation) or implicit (for e.g. in competitive multisite phosphorylation) $[24,26]$.

Although these two switch-like responses are generated by distinct classes of biochemical network designs these systems have several functional features in common. Both display high local sensitivity in their signal-response relationship (dLog[]/dLog[S]>>1) around a threshold signal level. Both can convert noisy unimodal input signals around the threshold into a distinctly bimodal response distribution (Panel B).The ultrasensitive systems and bistable systems also differ in some key functional properties. Two features that distinguish bistable networks are hysteresis (Panel B) and critical slowdown of the response around the signal threshold (Panel C). The hysteresis results from the multistability and results in history dependent response behavior - system may have multiple steady states corresponding to the same signal (shaded region in Panel B). In addition, the positive feedback makes their switching rate dependent on the signal strength $[26,28]$. As a result, response of bistable systems to sudden increase in signals much slowerespecially around the switching threshold (Panel $\mathrm{C}$ ). These properties can be advantageous by providing noise filtering capability and memory $[26,28]$, but can also be a hindrance when fast response times are crucial. These considerations can provide the rationale for the selection of bistable vs ultrasensitive networks to create switch-like response for differentiation GRNs. 
[22,23] (Box 1). Both ultrasensitive and bistable systems convert continuous signals into all-ornoneresponses based on a signal threshold [24-26]. However, unique dynamical properties of each mechanism can explain their selection for a particular differentiation program (Box 1).

Ability of differentiation GRNs such as the sporulation network to achieve switch-like behavior is usually easily demonstrated from the distinctly bimodal pattern of differentiation-specific genes downstream of the master regulator (e.g., $\sigma^{\mathrm{F}}$-controlled genes spollQ or $\sigma^{\mathrm{E}}$-controlled spolID [29] $\sigma^{\mathrm{G}}$-controlled sspB in sporulation). Moreover engineered strains, in which the master regulator activity is artificially controlled, can determine if a threshold activity is essential for differentiation commitment. Indeed, a threshold amount of SpoOA P is required for sporulation [31,32]. But which thresholding mechanism ultrasensitivity or bistability - is the basis for the all-or-none pattern of sporulation activation? Furthermore it is unclear whether this pattern originates at the level of the master regulator activity (e.g., SpoOA P) or those in the downstream signal-decoding modules (e.g., $\sigma^{\mathrm{F}}$ or $\sigma^{\mathrm{E}}$ activation).

For sporulation, several earlier studies hypothesized that the positive feedback loops in the phosphorelay network (Fig. 1B) make it into a bistable switch resulting in bimodal patterns of SpoOA activity [33]. This would explain the observed all-or-none sporulation commitment response. However subsequent single-cell measurements ruled out this hypothesis by demonstrating broad and unimodal distributions of Spo0A activity with significant overlap between sporulating and non-sporulating cells [34]. Mathematical modeling suggests that the phosphorelay operating as bistable switch would be very slow to activate potentially hindering successful sporulation [29]. This result offers a possible reason why bistable design wasn't evolutionarily selected in the case of sporulation.

The exclusion of SpoOA P bistability led into further inquiry whether activation of the $\sigma^{F}$ and $\sigma^{E}$ modules can explain the robust threshold of SpoOA activity and the all-or-none expression of downstream sporulation genes [29]. Mathematical modeling of activation of $\sigma^{F}$ and $\sigma^{E}$ modules showed that each of these modules respond ultrasensitively to changes in Spo0A P $[29,35]$. In the case of $\sigma^{F}$, the formation of a dead-end complex between the SpollAB (anti- $\sigma^{F}$ factor) and SpollAA (anti-anti- $\sigma^{F}$ factor) makes the ultrasensitive post-translational activation of $\sigma^{F}$ as a function of the level of SpoOA P-regulated phosphatase SpollE. For $\sigma^{\mathrm{E}}$ activation, the competition between Spo0A P-activated production of the unstable active form of $\sigma^{\mathrm{E}}$ and its rapid but saturable proteolytic degradation leads to an ultrasensitive response via a zero-order mechanism. Furthermore, $\sigma^{F}$ is controlled directly and indirectly by Spo0A $P$, forming a feedforward loop with one branch controlling $\sigma^{F}$ protein level and another one controlling its activation (Fig. 1B). Similarly, activation of $\sigma^{\mathrm{E}}$ is also controlled by SpoOA P both directly and indirectly (through $\sigma^{\mathrm{F}}$ ), forming another feed-forward loop. An integrated model of $\sigma^{\mathrm{F}}$ and $\sigma^{\mathrm{E}}$ activation predicted that the cascaded structure of these feed-forward loops combines the ultrasensitivity of the individual modules, resulting in a further increase in the sensitivity of sigma-factors activity to Spo0A P level (Fig. $1 C)$. This prediction was confirmed by investigating single-cell activity of SpoOA, $\sigma^{\mathrm{F}}$, and $\sigma^{\mathrm{E}}$ with a 3 distinct fluorescent reporters. With this strain, it was shown that, in single cells, the probability of activating downstream sigma factors increases ultrasensitively as a function of Spo0A activity (Fig. 1D). These experiments also confirmed another model prediction - that the threshold of SpoOA activity required for $\sigma^{\mathrm{F}}$ activation is less than that required for $\sigma^{\mathrm{E}}$ activation and consequently that $\sigma^{\mathrm{E}}$ activation is the point of sporulation commitment (Fig. 1C). Indeed, experiments with both wild-type and engineered strains showed that the fraction of cells activating $\sigma^{\mathrm{F}}$ exceeds the fraction activating $\sigma^{\mathrm{E}}$ and sporulating (Fig. 1D). Thus, $\sigma^{F}$ activation does not ensure completion of the sporulation program and therefore $\sigma^{\mathrm{E}}$ activation is the decision point in the sporulation network. 
When cell-fate decisions are made in decoding modules downstream of the master regulator, it allows the master regulator itself to be regulated more dynamically. This design enables master regulators like Spo0A P to encode more information about differentiation signals and the cellular environment than 1 bit maximally possible value for switch-like steady-state responses. As described below, investigations of the dynamics turn out to be critical for the sporulation network.

\section{Dynamic cell-cycle coupling}

Given that DNA replication and cell division are often essential for generation of different cell-types during differentiation, the underlying GRNs need to be coordinated with the cell cycle [2,36-39]. In some model systems, especially eukaryotes, the regulatory connections between cell-cycle and differentiation networks are well established [38-40]. Nevertheless, the detailed mechanisms of coupling between these are still incompletely understood. The problem arises because the cell-cycle by definition is a highly dynamic process. Accordingly, coordination with the cell-cycle requires the regulatory control to also be time-dependent. As a result, the standard approaches of understanding GRN function that focus on static diagrams of network structure fail to capture the underlying regulatory logic.

For sporulating $B$. subtilis, coordination of sporulation commitment with DNA replication is critically important [41]. Successful sporulation requires each compartment formed after asymmetric septation to quickly acquire its own chromosome in order to initiate the compartment specific sporulation gene expression programs [17]. Consequently, asymmetric cell division should only occur once DNA replication is complete (Fig. 2A). Since asymmetric septation is controlled by Spo0A P [42], this requirement can only be met by temporally coordinating SpoOA activation with DNA replication cycle. In line with this requirement, single-cell measurements of SpoOA activity revealed that it is indeed under dynamic control. In fact, recent experiments showed that during every cell cycle in starvation, Spo0A exhibits a single pulse of activity [43,44]. These Spo0A activity pulses always follow the completion of DNA replication [43-45]. Therefore, the sporulation network achieves coordination of SpoOA activation (and Spo0A P dependent septation) with completion DNA replication by using pulsatile activation.

The key to explaining Spo0A P pulsing and its cell-cycle coordination turns out to be a seemingly unrelated feature of the phosphorelay network. Mathematical model of the phosphorelay predicted that the inhibition of KinA autophosphorylation in the presence of excess phosphotransferase SpoOF - a feature of the phosphorelay that has been observed both in vitro [46] and in vivo [34,44] - plays a critical role in regulating Spo0A P dynamics [44]. First of all, since inhibition of KinA by SpoOF requires stoichiometric binding between the two, the phosphorylation flux can be very sensitive to the relative concentration of KinA and SpoOF. Second, the transcriptional activation of spoOF by SpoOA P together with the substrate inhibition results in a delayed negative feedback loop (Fig. 2B). Such network motifs are known to respond with pulsatile responses/overshoots $[47,48]$. Third, the spoOF gene is located near the origin of chromosome replication whereas kinA gene is near the terminus leading to transient imbalance in their production during replication. Taken together, the model predicted that KinA:SpoOF imbalance during replication would inhibit Spo0A activation. Following replication Spo0A P increases and due to the delayed negative feedback loop overshoots its steady state thereby producing a pulse (Fig. 2C). In other words - two design features of the network are required for pulsing - gene dosage imbalance and transcriptional feedback loop. 


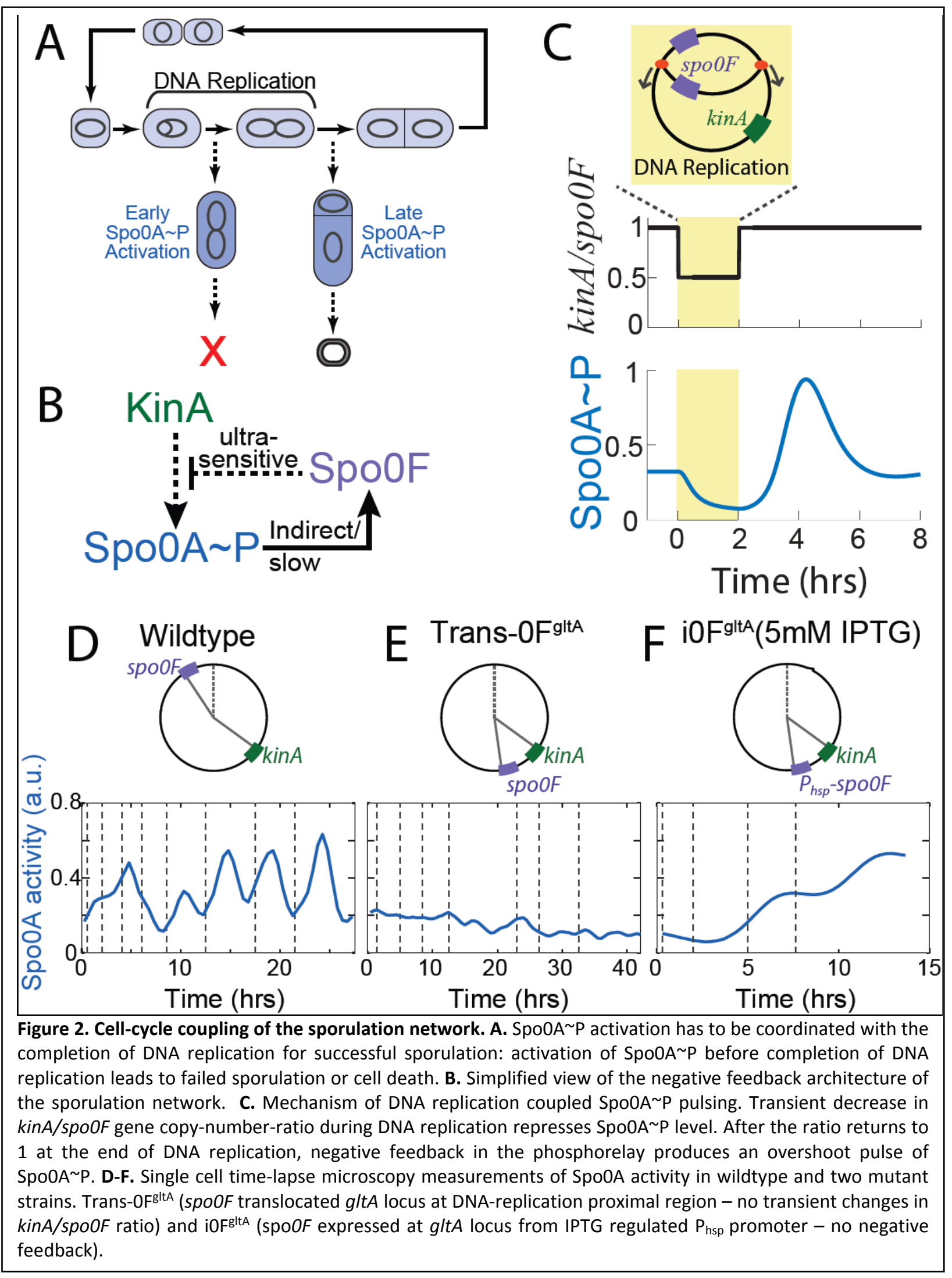


To test these predictions, single-cell measurements of Spo0A activity were performed in the wild-type strain as well as engineered strains in which the transcriptional feedback was eliminated by transcribing spoOF from inducible promoter and/or gene dosage imbalance was perturbed by translocation of either spoOF or kinA gene to a different locus (Fig. 2D-F). The results confirmed all model predictions Spo0A P pulsing, accumulation and consequently sporulation were eliminated in the strain in which spoOF was expressed from its native promoter at a terminus-proximal locus (Fig. 2E). However, if spoOF was expressed from the inducible promoter at low levels, starving cells show non-pulsatile increase in SpoOA activity and can eventually reach Spo0A-thereshold levels and sporulate (Fig. 2F). These observations raised an interesting question about the physiological role of pulses given that they are not absolutely required for sporulation. Careful analysis of single-cell phenotypes in time-lapse movies demonstrated that the engineered non-pulsatile strain showed a three-fold increase in sporulation defects. All the defects can be associated with attempts to commit to sporulation before completion of DNA-replication [44].

The major implication of these results is that GRNs can be implicitly coupled to cell cycle and DNA replication by proper chromosomal arrangement of network genes. This design feature can be exploited by differentiation GRNs when coupling is physiologically necessary. However, it can also unnecessarily entrain differentiation programs to chromosome duplication thereby making it essential for GRNs to include appropriate buffering mechanisms $[49,50]$. Notably, a different phosphorelay kinase, KinC can in certain genetic backgrounds bypass SpoOF [51] so activation of SpoOA by KinC may not be pulsatile. Given that this kinase that is active under conditions that do not produce spores, the same phosphorelay may be able to produce distinct dynamical responses activating alternative cell-fates. Such dynamic flexibility in the regulation of the master regulator may be a recurring feature for GRNs that control multiple differentiation programs. It should also be noted that many differentiation GRNs, including sporulation, are not only subject to regulation by the cell-cycle but also control the cell-cycle themselves [52]. Whether this type of mutual regulation enhances the robustness of coordination may be an interesting avenue for further studies.

\section{Signal integration and sensitivity to cell growth}

The traditional paradigm for understanding cell-fate decisions in cellular differentiation assumes that cells evaluate a signaling molecule (e.g. morphogen) concentration and respond with activation of certain gene expression programs. In many cases, such responses are assumed to involve a sensor protein that specifically binds the signaling molecule and transduces this information to downstream network components. This view tends to isolate the differentiation program from the milieu of metabolic, growth, and other cell-cycle processes within which it necessarily operates. However, variation in intrinsic physiological parameters such as growth rate is a major contributor to gene expression variability [53] and cell-fate heterogeneity [54,55]. Despite this evidence, we lack a clear understanding of how differentiation GRNs handle perturbations in physiological processes such as cell growth.

This deficiency is especially apparent for stress-activated differentiation programs which operate under conditions of suboptimal cell growth. Sporulation is a classic model of stress-activated programs. Starvation in B. subtilis can be triggered by a wide variety of environmental perturbations that cannot be defined by the level of a single metabolite $[16,56,57]$. To appropriately respond to starvation, the 
sporulation network must therefore solve two problems. First, it must be able to detect the availability of a variety of nutrients and integrate this information into the cellular response. Second, it has to be able to properly operate despite the inevitable consequences of starvation: slowdown of cell growth and its effects on gene expression and cell cycle.

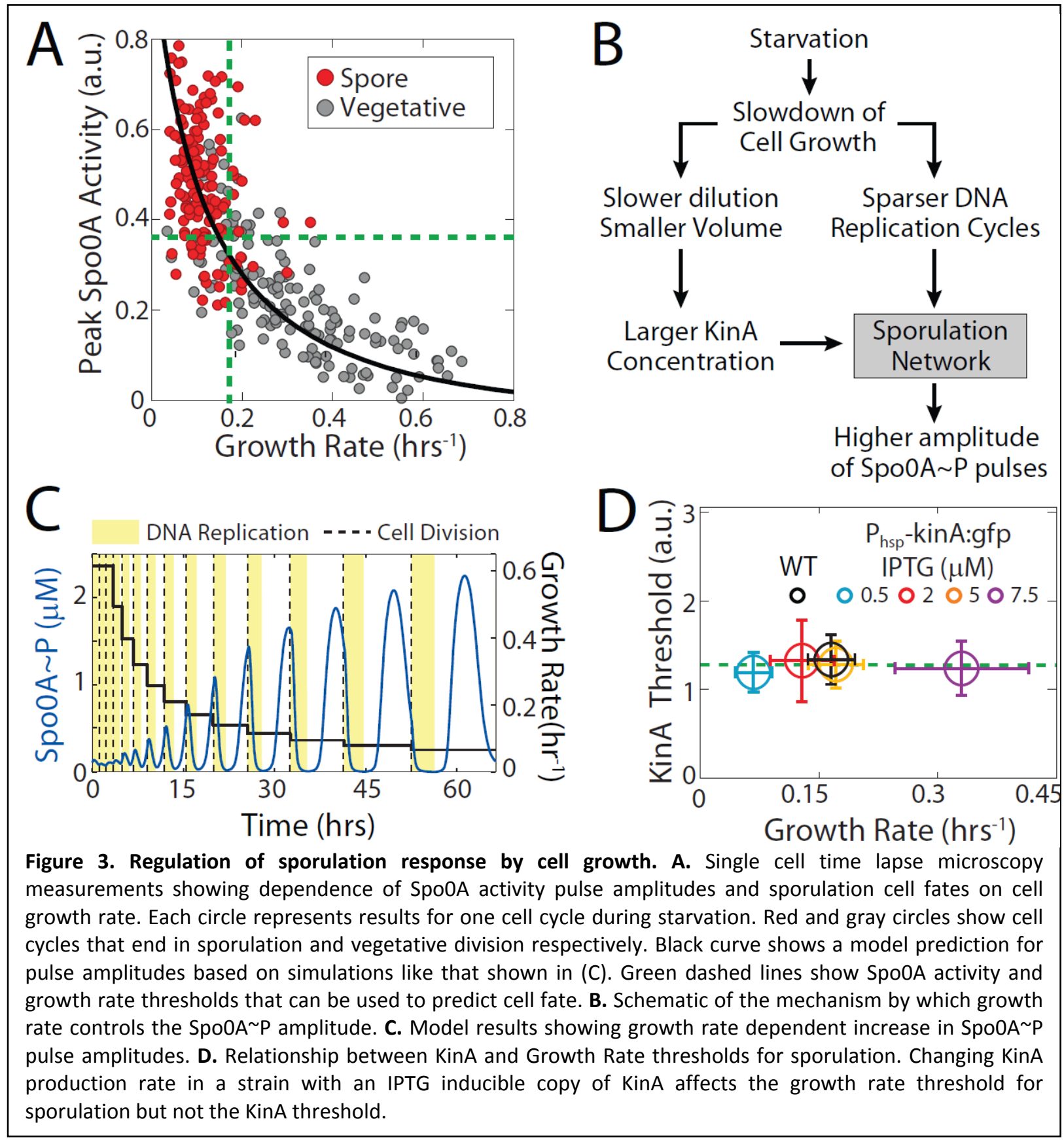

Prior studies of $B$. subtilis sporulation postulated that the design of the phosphorelay network offers multiple entry points for putative signals, e.g. via multiple kinases and phosphatases $[16,58,59]$. Consequently, the focus was identification of specific signaling molecules that regulate the phosphorelay response. In contrast, recently, a completely different hypothesis was investigated - whether the 
sporulation network can actually exploit one starvation related problem, i.e. growth-slowdown-related changes in gene expression, to solve another problem, i.e. integration of multiple nutrient levels into a single starvation response [60].

This hypothesis was motivated by single-cell time-lapse microscopy results showing that the amplitude of Spo0A P pulses is inversely correlated with cell growth (Fig. 3A). Furthermore, fates of over $85 \%$ cells can be explained by a simple model postulating that only cells growing slower than a threshold value sporulate (Fig. 3A). The same model also correctly predicted the distribution of sporulation deferral times during starvation based solely on the growth-rate distribution in each generation. But how can the sporulation network detect the cell growth rate?

To uncover a plausible mechanistic relationship between the growth rate and sporulation, the mathematical model of phosphorelay was used to uncover two complementary mechanisms (Fig. 3B). The model showed that as cell growth decreases, the cellular concentration of the rate-limiting phosphorelay kinase KinA increases as its dilution slows down and cell volume decreases. In addition, slowdown of growth leads to a longer time of DNA replication and also longer intervals between subsequent rounds of replication. Both effects can be quantified from the single-cell data and incorporated into the mathematical model of Spo0A P pulsing. The resulting model shows amplitudemodulation of SpoOA P comparable to that of the experiments (Fig. 3A, C). Furthermore, experiments with engineered strain, in which GFP-fused KinA is induced from an IPTG-inducible promoter, demonstrated that increasing KinA production can trigger sporulation in faster growing (less starving) cells. However, regardless of the starvation conditions the single-cell amount of KinA required for sporulation remains the same (Fig.3D). Therefore, in the conditions tested, KinA activity is not starvation-dependent and plays no role in controlling sporulation. Thus, in these experiments, growthslowdown-controlled KinA level is the primary starvation signal. On the other hand, under different conditions, additional signals that may also affect the phosphorelay and either serve as checkpoints prohibiting sporulation in certain conditions or modulating the growth-rate threshold required for sporulation.

The critical advantage of using cell growth as a starvation signal is that it is a naturally modulated by cellular metabolism. As such, cell growth integrates all the necessary nutrient information relevant for sporulation into a single signal. Consequently, growth sensitivity of the phosphorelay turns out to be a useful design feature of sporulation network. In contrast, for other stress-related differentiation programs, growth-rate sensitivity may represent a major functional challenge. Recent studies have demonstrated how changes in cell growth can impact the partitioning of cellular resources such as RNA polymerase and ribosomes [61-63] that are shared among all cellular processes including differentiation. Understanding the impact of cell growth on differentiation GRNs for a wide range of model systems represents a major direction for future studies of cellular differentiation.

\section{Conclusions}

The remarkable complexity and diversity of differentiation GRNs represents a daunting obstacle to our understanding of their functionality. We hope that the examples from the studies of sporulation illustrate how a combination of mathematical modeling and single-cell experiments can be used to tackle these networks. Critically, modeling enables the systematic reduction of complexity by 
abstraction of networks by uncovering their core functional structure (e.g., phosphorelay as a delayed negative feedback loop; $\sigma^{\mathrm{E}}$ activation network as a zero-order ultrasensitivity module). From this perspective, it becomes far easier to relate the network design to functional properties and requirements (e.g. cell-cycle coordination by the pulse-generating negative feedback loop or binary cellfate decisions by the ultrasensitive $\sigma^{F}$ and $\sigma^{E}$ activation networks). These approaches also enable the identification of critical design features that are rarely evident on a network wiring diagram, such as chromosomal gene arrangement and growth sensitivity, which could be recurrent features across different differentiation programs with seemingly little in common.

\section{Acknowledgements:}

This work is supported by National Science Foundation grants MCB-1244135 (to OAI) and MCB-1244423 (to MF).

\section{References}

1. Slack JMW: Essential developmental biology edn 3rd. Chichester, West Sussex; Hoboken, NJ: Wiley; 2013.

2. Davidson EH: Emerging properties of animal gene regulatory networks. Nature 2010, 468:911-920.

3. Davidson EH, Levine MS: Properties of developmental gene regulatory networks. Proc Natl Acad Sci U S A 2008, 105:20063-20066.

4. England JC, Perchuk BS, Laub MT, Gober JW: Global regulation of gene expression and cell differentiation in Caulobacter crescentus in response to nutrient availability. J Bacteriol 2010, 192:819-833.

5. Lopez D, Vlamakis H, Kolter R: Generation of multiple cell types in Bacillus subtilis. FEMS Microbiol Rev 2009, 33:152-163.

6. Palkova Z, Wilkinson D, Vachova L: Aging and differentiation in yeast populations: elders with different properties and functions. FEMS Yeast Res 2014, 14:96-108.

7. Loomis WF: Genetic control of morphogenesis in Dictyostelium. Dev Biol 2015, 402:146-161.

8. Davidson EH: The regulatory genome: gene regulatory networks in development and evolution. Amsterdam ; Boston: Elsevier/Academic Press; 2006.

9. Eser U, Falleur-Fettig M, Johnson A, Skotheim JM: Commitment to a cellular transition precedes genome-wide transcriptional change. Mol Cell 2011, 43:515-527.

10. Fujita M, Gonzalez-Pastor JE, Losick R: High- and low-threshold genes in the SpoOA regulon of Bacillus subtilis. J Bacteriol 2005, 187:1357-1368.

11. Ptashne M: A genetic switch : phage lambda revisited edn 3rd. Cold Spring Harbor, N.Y.: Cold Spring Harbor Laboratory Press; 2004.

12. Levine M, Davidson EH: Gene regulatory networks for development. Proc Natl Acad Sci U S A 2005, 102:4936-4942.

13. de Hoon MJ, Eichenberger P, Vitkup D: Hierarchical evolution of the bacterial sporulation network. Curr Biol 2010, 20:R735-745.

14. Eichenberger P, Fujita M, Jensen ST, Conlon EM, Rudner DZ, Wang ST, Ferguson C, Haga K, Sato T, Liu $J S$, et al.: The program of gene transcription for a single differentiating cell type during sporulation in Bacillus subtilis. PLoS Biol 2004, 2:e328.

15. Higgins D, Dworkin J: Recent progress in Bacillus subtilis sporulation. FEMS Microbiol Rev 2012, 36:131148. 
16. Hoch JA: Regulation of the phosphorelay and the initiation of sporulation in Bacillus subtilis. Annu Rev Microbiol 1993, 47:441-465.

17. Hilbert DW, Piggot PJ: Compartmentalization of gene expression during Bacillus subtilis spore formation. Microbiol Mol Biol Rev 2004, 68:234-262.

18. Wang ST, Setlow B, Conlon EM, Lyon JL, Imamura D, Sato T, Setlow P, Losick R, Eichenberger P: The forespore line of gene expression in Bacillus subtilis. J Mol Biol 2006, 358:16-37.

19. Alon U: Network motifs: theory and experimental approaches. Nat Rev Genet 2007, 8:450-461.

20. Stathopoulos A, Levine M: Genomic regulatory networks and animal development. Dev Cell 2005, 9:449-462.

21. Wall ME, Hlavacek WS, Savageau MA: Design of gene circuits: lessons from bacteria. Nat Rev Genet 2004, 5:34-42.

22. Becskei A, Seraphin B, Serrano L: Positive feedback in eukaryotic gene networks: cell differentiation by graded to binary response conversion. EMBO J 2001, 20:2528-2535.

23. Xiong W, Ferrell JE, Jr.: A positive-feedback-based bistable 'memory module' that governs a cell fate decision. Nature 2003, 426:460-465.

24. Ferrell JE, Jr., Ha SH: Ultrasensitivity part III: cascades, bistable switches, and oscillators. Trends Biochem Sci 2014, 39:612-618.

25. Ferrell JE, Jr., Ha SH: Ultrasensitivity part I: Michaelian responses and zero-order ultrasensitivity. Trends Biochem Sci 2014, 39:496-503.

26. Tiwari A, Ray JC, Narula J, Igoshin OA: Bistable responses in bacterial genetic networks: designs and dynamical consequences. Math Biosci 2011, 231:76-89.

27. Ray JC, Tabor JJ, Igoshin OA: Non-transcriptional regulatory processes shape transcriptional network dynamics. Nat Rev Microbiol 2011, 9:817-828.

28. Narula J, Williams CJ, Tiwari A, Marks-Bluth J, Pimanda JE, Igoshin OA: Mathematical model of a gene regulatory network reconciles effects of genetic perturbations on hematopoietic stem cell emergence. Dev Biol 2013, 379:258-269.

29. Narula J, Devi SN, Fujita M, Igoshin OA: Ultrasensitivity of the Bacillus subtilis sporulation decision. Proceedings of the National Academy of Sciences of the United States of America 2012, 109:E3513-E3522.

*This paper provides evidence that the sporulation decision is made downstream of SpoOA P by a cascade of coherent feed-forward loops, that ultrasensitively control $\sigma^{F}$ and $\sigma^{E}$ activation. Furthermore, it shows that SpoOA and $\sigma^{F}$ activation are reversible whereas $\sigma^{\mathrm{E}}$ activation controls irreversible commitment to sporulation.

30. Vlamakis H, Aguilar C, Losick R, Kolter R: Control of cell fate by the formation of an architecturally complex bacterial community. Genes Dev 2008, 22:945-953.

31. Chung JD, Stephanopoulos G, Ireton K, Grossman AD: Gene expression in single cells of Bacillus subtilis: evidence that a threshold mechanism controls the initiation of sporulation. J Bacteriol 1994, 176:1977-1984.

32. Eswaramoorthy P, Duan D, Dinh J, Dravis A, Devi SN, Fujita M: The threshold level of the sensor histidine kinase KinA governs entry into sporulation in Bacillus subtilis. J Bacteriol 2010, 192:38703882.

*This paper uses an engineered strain to show that inducing the synthesis of the phosphorelay kinase KinA beyond a certain level leads to sporulation initiation irrespective of nutrient availability. 
33. Veening JW, Hamoen LW, Kuipers OP: Phosphatases modulate the bistable sporulation gene expression pattern in Bacillus subtilis. Mol Microbiol 2005, 56:1481-1494.

34. Chastanet A, Vitkup D, Yuan GC, Norman TM, Liu JS, Losick RM: Broadly heterogeneous activation of the master regulator for sporulation in Bacillus subtilis. Proc Natl Acad Sci U S A 2010, 107:84868491.

*This paper reinvestigates the hypothesis that activation of Spo0A and hence entry into sporulation is subject to a bistable switch. Genes under direct Spo0A control are shown to exhibit a heterogeneous but non-bimodal pattern of expression ruling out the idea that the phosphorelay is a bistable switch.

35. Igoshin OA, Price CW, Savageau MA: Signalling network with a bistable hysteretic switch controls developmental activation of the sigma transcription factor in Bacillus subtilis. Mol Microbiol 2006, 61:165-184.

36. Kim YH, Larsen HL, Rue P, Lemaire LA, Ferrer J, Grapin-Botton A: Cell cycle-dependent differentiation dynamics balances growth and endocrine differentiation in the pancreas. PLoS Biol 2015, 13:e1002111.

37. Kishi H, Nakagawa K, Matsumoto $M$, Suga $M$, Ando $M$, Taya $Y$, Yamaizumi M: Osmotic shock induces G1 arrest through p53 phosphorylation at Ser33 by activated p38MAPK without phosphorylation at Ser15 and Ser20. J Biol Chem 2001, 276:39115-39122.

38. Levin PA, Grossman AD: Cell cycle and sporulation in Bacillus subtilis. Curr Opin Microbiol 1998, 1:630635.

39. Li VC, Kirschner MW: Molecular ties between the cell cycle and differentiation in embryonic stem cells. Proceedings of the National Academy of Sciences of the United States of America 2014, 111:95039508.

40. Abel S, Chien P, Wassmann P, Schirmer T, Kaever V, Laub MT, Baker TA, Jenal U: Regulatory cohesion of cell cycle and cell differentiation through interlinked phosphorylation and second messenger networks. Mol Cell 2011, 43:550-560.

41. Ireton K, Grossman AD: Coupling between gene expression and DNA synthesis early during development in Bacillus subtilis. Proc Natl Acad Sci U S A 1992, 89:8808-8812.

42. Ben-Yehuda S, Losick R: Asymmetric cell division in B. subtilis involves a spiral-like intermediate of the cytokinetic protein FtsZ. Cell 2002, 109:257-266.

43. Levine JH, Fontes ME, Dworkin J, Elowitz MB: Pulsed feedback defers cellular differentiation. PLoS Biol 2012, 10:e1001252.

* Quantitative time-lapse fluorescence microscopy of SpoOA dynamics in individual cells reveals that pulses of SpoOA phosphorylation occur at a characteristic cell cycle phase. In addition systematic growth of pulse amplitudes over multiple cell cycles is shown to be essential for sporulation deferral.

44. Narula J, Kuchina A, Lee DY, Fujita M, Suel GM, Igoshin OA: Chromosomal Arrangement of Phosphorelay Genes Couples Sporulation and DNA Replication. Cell 2015, 162:328-337.

** This paper demonstrates that the chromosomal arrangement of two sporulation network genes spoOF (origin proximal) and kinA (terminus proximal), creates a transient gene dosage imbalance during DNA replication. This imbalance is detected and amplified by a negative feedback in the phosphorelay to produce cell-cycle coordinated pulses of the sporulation master regulator Spo0A P.

45. Veening J-W, Murray $\mathrm{H}$, Errington J: A mechanism for cell cycle regulation of sporulation initiation in Bacillus subtilis. Genes Dev 2009, 23:1959-1970. 
46. Grimshaw CE, Huang S, Hanstein CG, Strauch MA, Burbulys D, Wang L, Hoch JA, Whiteley JM: Synergistic kinetic interactions between components of the phosphorelay controlling sporulation in Bacillus subtilis. Biochemistry 1998, 37:1365-1375.

47. Ma W, Trusina A, El-Samad H, Lim WA, Tang C: Defining network topologies that can achieve biochemical adaptation. Cell 2009, 138:760-773.

48. Ascensao JA, Datta P, Hancioglu B, Sontag E, Gennaro ML, Igoshin OA: Non-monotonic Response to Monotonic Stimulus: Regulation of Glyoxylate Shunt Gene-Expression Dynamics in Mycobacterium tuberculosis. PLoS Comput Biol 2016, 12:e1004741.

49. Paijmans J, Bosman M, Ten Wolde PR, Lubensky DK: Discrete gene replication events drive coupling between the cell cycle and circadian clocks. Proc Natl Acad Sci U S A 2016, 113:4063-4068.

50. Voichek Y, Bar-Ziv R, Barkai N: Expression homeostasis during DNA replication. Science 2016, 351:10871090.

** Evidence that in budding yeast H3K56 acetylation plays an essential role in buffering against changes in gene dosage during $\mathrm{S}$ phase and preventing differences in mRNA synthesis rates of earlyreplicating and late-replicating genes

51. LeDeaux JR, Grossman AD: Isolation and characterization of kinC, a gene that encodes a sensor kinase homologous to the sporulation sensor kinases KinA and KinB in Bacillus subtilis. J Bacteriol 1995, 177:166-175.

52. Boonstra M, de Jong IG, Scholefield G, Murray H, Kuipers OP, Veening JW: SpoOA regulates chromosome copy number during sporulation by directly binding to the origin of replication in Bacillus subtilis. Mol Microbiol 2013, 87:925-938.

53. Kiviet DJ, Nghe P, Walker N, Boulineau S, Sunderlikova V, Tans SJ: Stochasticity of metabolism and growth at the single-cell level. Nature 2014, 514:376-379.

*This work provides about possible insights into the spurce of stochasticity in single cell growth rates - it can be caused by fluctuations in rate-limiting enzymes.

54. Snijder B, Pelkmans L: Origins of regulated cell-to-cell variability. Nat Rev Mol Cell Biol 2011, 12:119125.

55. St-Pierre F, Endy D: Determination of cell fate selection during phage lambda infection. Proc Natl Acad Sci U S A 2008, 105:20705-20710.

56. Dawes IW, Mandelstam J: Sporulation of Bacillus subtilis in continuous culture. J Bacteriol 1970, 103:529-535.

57. Ochi K, Kandala J, Freese E: Evidence that Bacillus subtilis sporulation induced by the stringent response is caused by the decrease in GTP or GDP. J Bacteriol 1982, 151:1062-1065.

58. Ireton K, Rudner DZ, Siranosian KJ, Grossman AD: Integration of multiple developmental signals in Bacillus subtilis through the SpoOA transcription factor. Genes Dev 1993, 7:283-294.

59. Trach KA, Hoch JA: Multisensory activation of the phosphorelay initiating sporulation in Bacillus subtilis: identification and sequence of the protein kinase of the alternate pathway. Mol Microbiol 1993, 8:69-79.

60. Narula J, Kuchina A, Zhang F, Fujita M, Suel GM, Igoshin OA: Slowdown of growth controls cellular differentiation. Mol Syst Biol 2016, 12:871.

This paper shows that the activity of sporulation master regulator SpoOA increases with decreasing cellular growth rate and only cells growing slower than a certain rate reach threshold SpoOA activity necessary for sporulation. Further it is shown that this increase in SpoOA activity can be explained by the phosphorelay protein accumulation and lengthening of the period between chromosomal replication events caused by growth slowdown. 
61. Klumpp S, Zhang Z, Hwa T: Growth rate-dependent global effects on gene expression in bacteria. Cell 2009, 139:1366-1375.

62. Borkowski O, Goelzer A, Schaffer M, Calabre M, Mader U, Aymerich S, Jules M, Fromion V: Translation elicits a growth rate-dependent, genome-wide, differential protein production in Bacillus subtilis. Mol Syst Biol 2016, 12:870.

*This paper demonstrates how growth rate increase in B. subtilis is associated with a decrease in free ribosome abundance resulting in the decrease in translation efficiencies and protein production with increasing growth rate.

63. Hui S, Silverman JM, Chen SS, Erickson DW, Basan M, Wang J, Hwa T, Williamson JR: Quantitative proteomic analysis reveals a simple strategy of global resource allocation in bacteria. Mol Syst Biol 2015, 11:784. 


\section{Graphical_Abstract (for review)}

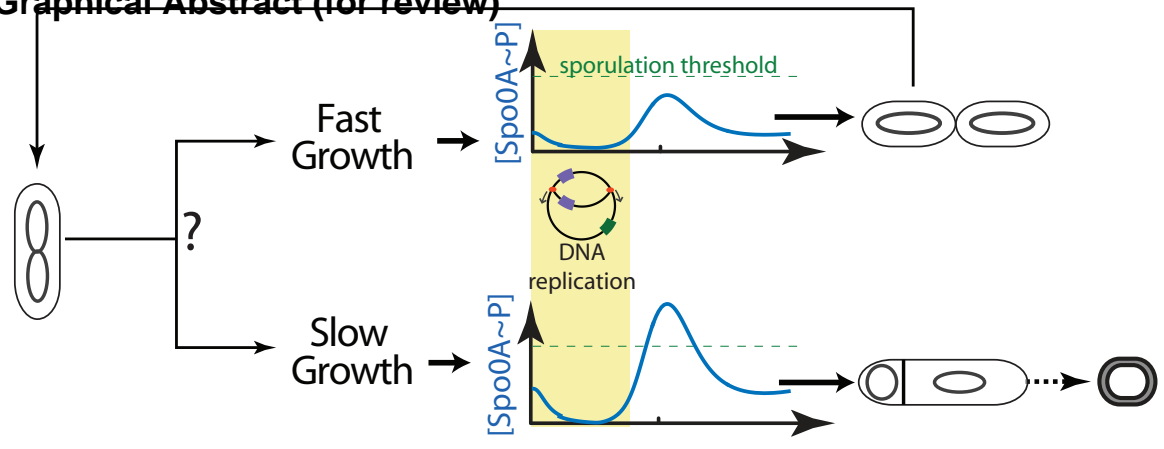

